\title{
Molecular Materials for Energy Storage
}

\author{
Lev Mourokh ${ }^{1,2,3}$, Carine Edder ${ }^{1}$, Wolfgang Mack ${ }^{1}$, Pavel Lazarev1 \\ ${ }^{1}$ Capacitor Sciences Inc., Menlo Park, USA \\ ${ }^{2}$ Department of Physics, Queens College of the City, University of New York, Flushing, USA \\ ${ }^{3}$ The Graduate Center of CUNY, New York, NY, USA \\ Email:1ev.murokh@qc.cuny.edu
}

How to cite this paper: Mourokh, L., Edder, C., Mack, W. and Lazarev, P. (2018) Molecular Materials for Energy Storage. Materials Sciences and Applications, 9, 517-525.

https://doi.org/10.4236/msa.2018.96036

Received: March 29, 2018

Accepted: May 25, 2018

Published: May 28, 2018

Copyright $\odot 2018$ by authors and Scientific Research Publishing Inc. This work is licensed under the Creative Commons Attribution International License (CC BY 4.0). http://creativecommons.org/licenses/by/4.0/

\begin{abstract}
We map the requirements and design rules for dielectric materials that target large scale energy storage applications of electrostatic capacitors. The molecular unit (dielectrophore) must contain three main components: a polarizable subunit having large electric dipole, an isolating subunit which prevents current leakage, and a structural element that promotes self-assembly of molecules in solution and in mesophase and promotes crystallization in the process of film formation. This structural subunit is necessary for high molecular density and enhanced resistivity of film. The process engineering steps required for the supramolecular assembly and crystal film formation (cascade crystallization) are addressed.
\end{abstract}

\section{Keywords}

Capacitor, Molecular Dielectrics, Molecular Design, Polarization, Induced Electric Dipole

\section{Introduction}

Electric energy storage is a key component for the next stage of human civilization. We have now entered the early stages of the fully-electric economy including home, transportation, communications, entertainment and industrial operations. Transportation is undergoing a visible transformation from that of internal combustion engines burning fossil fuels to that of electric motors driven by electrical energy. The proliferation of renewable energy also requires energy storage to balance generation and consumption. As a result, we have seen the rapid deployment of energy storage schemes including flywheels, compressed air, heavy weighs on rails and various forms of batteries primarily in utility, commercial and industrial markets. The Tesla Powerwall sparked demand for residential energy storage, promoting the need to store solar energy and to charge 
electric vehicles. Each of the existing energy storage technologies has limitations that prevent global-scale deployment to support this shift toward the fully-electric economy. A new approach is needed to create energy storage devices that can store electrical energy for home, vehicular, commercial and industrial applications.

The scale of production of energy storage materials required to store globally consumed electricity can be understood with the following analysis. Assuming an energy storage device with a specific energy of $1 \mathrm{kWh} / \mathrm{kg}$, one can determine the amount of material required to store 24 hours of globally consumed electricity. The U.S. Energy Information Administration (EIA) estimated global electricity production to surpass 25,000 billion $\mathrm{kWh}$ in 2020 growing to over 35,000 billion kWh in 2040 [1].

- 25,000 billion $\mathrm{kWh} /$ year divided by 365 days/year = 68 billion $\mathrm{kWh} /$ day;

- 68 billion $\mathrm{kWh} /$ day divided by $1 \mathrm{kWh} / \mathrm{kg}=68$ billion $\mathrm{kg}$ of energy storage materials;

- 68 billion $\mathrm{kg} / 1000 \mathrm{~kg} / \mathrm{tonne}=68$ million tonnes of energy storage materials;

- 68 million tonnes $/ 10$ years $=6.8$ million tonnes per year of energy storage materials;

- Rounding up yields 10 million tonnes per year of $1 \mathrm{kWh} / \mathrm{kg}$ energy storage materials.

The scale of energy storage material production required for 24 hours of global electricity consumption can now be compared with the global production of commodity materials that could also be used for energy storage. Following are commodity materials and their respective annual production:

- Global electricity storage $1 \mathrm{kWh} / \mathrm{kg}$ material requirement: 10 million tonnes per year;

- Polyethylene: 80 million tonnes per year;

- Polyethylene Terephthalate (PET): 30 million tonnes per year;

- Polypropylene (PP): 1 million tonnes per year;

- Lithium: 0.047 million tonnes per year (300 million tonnes of lithium per year required).

In order to provide for global energy storage, lithium production must increase by at almost 4 orders of magnitude to meet the required production demand. Polyethylene and polypropylene are produced in the quantities required to provide materials on the scale of 10 million tonnes per year needed to deploy global energy storage, however, an equivalent global manufacturing capacity will need to be developed. The only raw material able to support the required $10 \mathrm{mil}-$ lion tonnes per year production capacity for global energy storage is oil. It is inexpensive, abundant and easily modified to produce high specific energy metadielectric materials at the scale required for global electricity storage.

Presently, the main storage devices are batteries. Energy is stored electrochemically and electrons released during chemical reactions exit the battery and enter electric circuits to perform work. The energy density of batteries is sufficient for many applications; however, they are relatively expensive about 
$\$ 100 / \mathrm{kWh}$ at the cell level with cost reductions forecast to $\$ 50 / \mathrm{kWh}$ by 2025 . During the charging process, the carriers (like lithium ions) are returned back to the source. This process is slow and, moreover, it degrades the battery materials. Thus, the power density of the batteries is low, and their lifetime is short due to the material damage. Furthermore, the batteries become less reliable at elevated temperatures. Alternative storage devices are capacitors, where the energy is stored electrostatically. The charging process is fast, leading to the high-power density and the longtime reliability. Capacitors have the advantages over the batteries in almost all areas but currently have much lower energy density [2]. In order to overcome this drawback, novel devices called supercapacitors have been developed [3]. They have energy densities greater than those of film capacitors and power densities greater than those of batteries. However, there is still no device having the power density comparable to film capacitors and the energy density comparable to batteries. Moreover, the cost of supercapacitors remains relatively high.

Energy storage devices having both high energy density and high-power density are crucial for electric vehicle and stationary power applications. As the limitations of electrochemical batteries are impossible to overcome the high energy density and high-power density solution should be that of a high energy density film capacitor. In this paper, we propose a class of metadielectric materials that can provide this breakthrough and lead to inexpensive, reliable film capacitors to overcome the deficiencies of electrochemical energy storage devices.

Our paper is structured as follows. In Section II, we state the main principles of molecular design to achieve the highest induced polarization and highest charge retention. In Section III, we propose employing cascade crystallization to form ordered dielectric films from the proposed metadielectric materials. Section IV presents the conclusions of our work.

\section{Molecular Design for Energy Storage Applications}

The dielectric film for capacitors should satisfy several requirements. First, the film must be polarizable. An applied electric field must induce dipoles (or align existing ones) to create the polarization serving as the energy store. Second, conduction current inside the material should be minimal. Moreover, the material must sustain operational voltage without breakdown and maintain its specified dielectric strength. These two properties seem contradictory, as the polarization is caused by the motion of the charges, but this motion has to be limited to prevent conduction leading to charge recombination at the electrode. Consequently, molecular units, which we call dielectrophores, should contain at least two parts, one being responsible for the polarization, and the second one having required resistance.

The operation of a film capacitor requires even distribution of the external field and uniformity of the dielectrophore film material in order to create an 
even distribution of the induced counter-field within capacitor. The best structure of material with uniform distribution of properties is a crystal. It might also be advisable to include a structural element in the molecule that would promote the crystal structure of the film.

It is important to appreciate the fact that capacitors are high pressure devices with pressure developed by the Coulomb attraction of oppositely charged electrodes. In order for capacitive devices to remain operable the elasticity of dielectric material layer must also be uniform. In other words, elements of a composite material dielectric film need to be of approximately the same plasticity and strength in order to transfer pressure in all directions similar to a liquid material.

The structural element can be added to promote the high-density supramolecular self-assembly and the formation of a structured, crystalline dielectric film. The resulting general structure of the dielectrophore is shown in Figure 1(a). With an applied electric field in horizontal direction, the electric dipole will be formed within the polarizable subunit, while the insulative tails will prevent charge transport to the next dielectrophore. The core subunit allows the supramolecular stacking which occurs in the direction perpendicular to the plane of the image.

Previously, ceramics, such as silicon oxide, silicon nitride and barium titanate, have been used as high dielectric constant materials [4]. However, for these composites the dielectric strength is low, they exhibit high dielectric loss (current leakage) and these materials are easily damaged when they are subjected to the high pressure associated with high operational voltage.

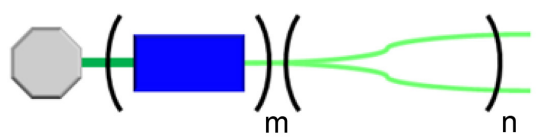

(a)

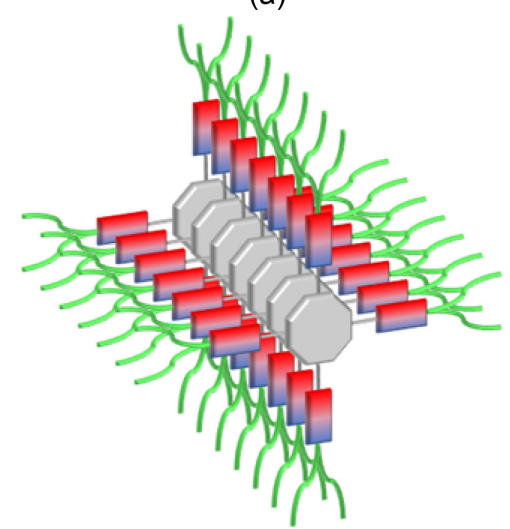

(b)

Figure 1. (a) The structure of the dielectrophore containing $m$ polarizable subunits (blue rectangular), $n$ subunits with high resistance (green tails), and a core (grey octagon) responsible for the stacking of dielectrophores into a column-like supramolecular assembly. (b) Supramolecular column formed by the $\pi-\pi$ stacking of the aromatic cores. 
Oil-derivative organic compounds are the most suitable basis for film capacitor designs for the following reasons. First, organic molecules allow the flexibility of design of the elementary units. Second, these molecules can form structured kinetic units required for self-assembly. Third, the $\pi-\pi$ stacking of aromatic molecules makes it possible to form highly dense self-assembled supramolecular columns. Fourth, the obtained supramolecular would be flexible enough not to be damaged by high operating pressure. And finally, the synthesized organic materials are relatively inexpensive and can be produced in the volumes necessary to satisfy the global demand for energy storage. While the use of oil for energy production will decrease in favor of renewable energy sources, its reserves are still formidable and can be used for organic synthesis of advanced functional materials including dielectrophores.

An example of proposed design is shown in Figure 1(b). The aromatic core allows for $\pi-\pi$ stacking and the formation of the supramolecular column. The polarizable subunits are attached to the core and the insulating tails are attached to the polarizable subunits. The insulating tails prevent charge transfer to the adjacent supramolecular columns eliminating current leakage (dielectric loss) and dielectric breakdown. These supramolecular columns would form in the plane perpendicular to the capacitor electrodes and isolated from each other in a flexible quasiperiodic order. The directions of polarizable units can be random or might be slightly ordered by the inter-unit electrostatic coupling. Supramolecular column alignment will be achieved under non-zero external electric field. In order to achieve high energy density, polarizable subunits must have sufficient ability to create induced electric dipoles in response to the external electric field that produce an internal counter field. The origin of polarizability can be different [5].

In neutral atoms, the displacement of the center of the negatively charged electron clouds from the positively charged nucleus produces electronic polarization. Ionic polarization is caused by the separation of positive and negative ions in molecules and crystals from their equilibrium positions by means of the external field. Both electronic and ionic polarizations are induced by an external electric field. For polar molecules, molecules with built-in permanent dipole moments, the application of an external field would result in their alignment. With no applied field, the dipole orientations are random and are averaged to produce a zero-dipole moment. In the presence of an external electric field, the dipole direction along the axis of the field is energetically favorable leading to dipolar or orientation polarization. If the dielectrophore material contains free charges, they can be displaced over a long distance from their host nuclei by the external field. This would lead to space-charge polarization. The analog of such an effect exists in large molecules and is referred to as hyperelectronic polarization [6]. It becomes pronounced at low frequencies as the charge displacement processes on the molecular scale are relatively slow. However, for the static response necessary for energy storage in capacitors, the hyperelectronic polarization is especially promising. 
For the purposes of energy storage, we propose to employ hyperelectronic and orientational polarization in the dielectrophores, as shown in Figure 2(a). When the external electric field is applied, electric dipoles are formed in the polarizable units. If the molecules are long enough these induced dipoles can be quite large due to hyperelectronic polarization. Polar molecules with built-in dipole moments aligned by the external field will achieve significant induced polarization of the dielectrics. The polarizable units are separated by the high resistance tails to prevent the current leakage. The resulting multilayered structure is shown in Figure 2(b). We believe that such capacitors would exhibit all three properties necessary for high density energy storage: high polarizability of a single unit, strong unit isolation leading to a low leakage and the supramolecular column structures resulting in high density of polarizable units.

\section{Cascade Crystallization}

The assembly of dielectrophore materials to form the structure illustrated in Figure 2(b) presents a significant challenge. First, the volume ratio of polarizable units and resistive tails should be optimal to achieve the highest polarization possible without decreasing the dielectric strength of the film. Second, the

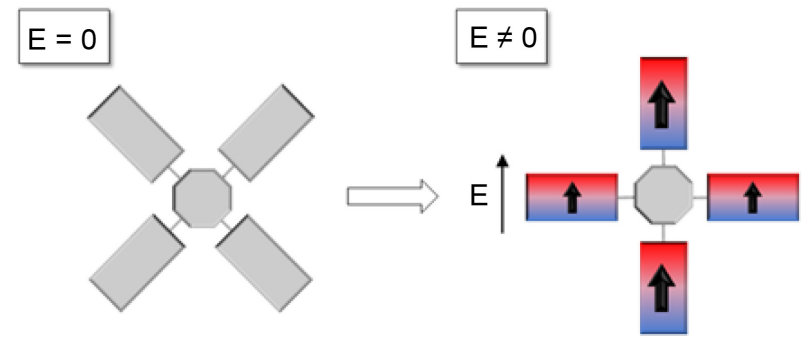

(a)

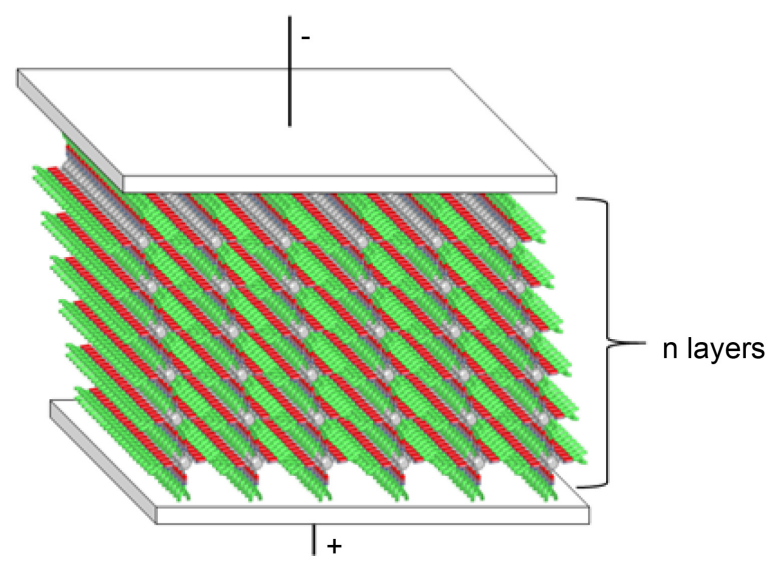

(b)

Figure 2. (a) Formation of polarization in the dielectrophores. The hyperelectronic polarization in the response to an external electric field leads to the creation of the electric dipole in the polarizable subunits. The orientation polarization would align the dipoles along the electric field. (b) Schematics of the capacitor filled by the multilayered structure allowing for both high polarization and high resistivity. 
molecular cores and polarizable subunits of supramolecular structures need to be aligned perpendicular to the plane of the electrodes. This should result in separated layers of stacked polarizable subunits and intertwined resistive groups, that would prevent excited electrons from conducting to adjacent supramolecular columns and eventually to the positive electrode. Third, the resulting crystal structure should not be too rigid, so that high voltage and associated high pressure would not compromise the supramolecular column order and lead to mechanical corrugation. Fourth, the structure should be able to assemble on any arbitrary substrate, as any additional layers added specifically for alignment and crystallization would reduce the density of polarizable subunits and reduce the specific energy of the device. The first two requirements can be satisfied by means of computation chemistry and a series of "probe and learn" experiments. The fourth condition would be naturally realized in the case of organic molecules with flexible unit arrangement and with the crystalline order not dependent on the substrate properties. To achieve that, we have proposed a procedure which we call cascade crystallization [7] [8]. Pre-ordered materials which form lyotropic liquid crystals with local crystalline order are used to avoid the effect of the electrode substrates on the crystal film growth and to arrange the polarizable cores perpendicular to the plan of the electrodes. The main steps of the cascade crystallization process are shown in Figure 3.

Indeed, to achieve optimal device performance of those materials within the capacitor, the molecules need to be oriented in a highly ordered spatial arrangement, to increase polarizability with optimal alignment of the polarizable subunits and prevent any conduction pathway between the electrode through the dielectric material. Cascade crystallization is a process that forms thin films of such ordered nano-structures. It consists of a molecular design step, and four steps of ordering during the crystal dielectric layer formation. The first step involves designing a molecule that contains polarizable units that would interact with each other by $\pi$-stacking. Long alkyl chains are introduced at the peripheries of the molecules to enable solubility, to suppress lateral aggregation in solution and to impart mesogenicity of the thin film material with interdigitation of the alkyl chains.

In the first step of ordering, the molecules self-assemble in solutions into one-dimensional stacks, by means of $\pi-\pi$ interactions, even at low concentration

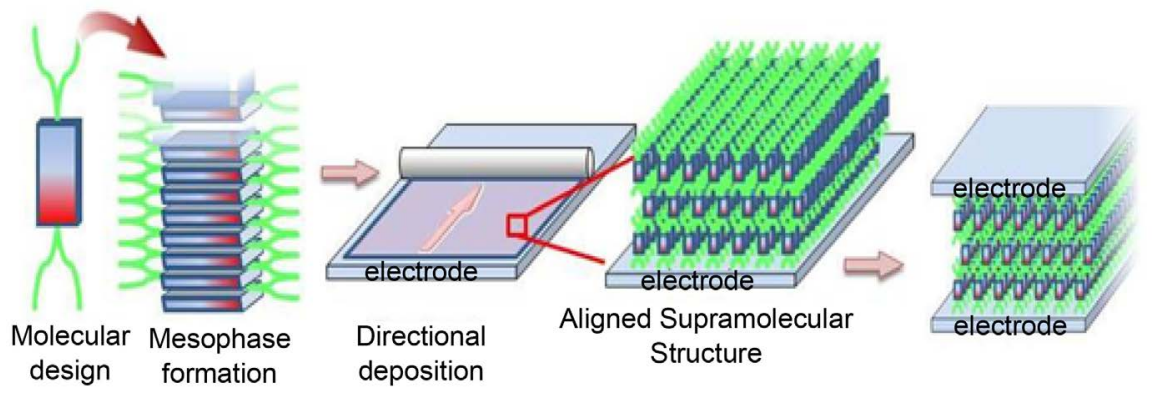

Figure 3. Steps of the cascade crystallization process. 
[9]. The molecular planar cores of aromatic conjugated bond systems are stacked on each other inside those supramolecular columns and the peripheral alkyl chains are exposed to the solvent. The interplanar spacing in the stack would be of the order of $3.4 \pm 0.3 \AA$. At a certain concentration, the supramolecular structures reach a liquid-crystalline state to form a lyotropic liquid crystal [7] [8] [10] which is the second step of ordering.

This induced liquid crystallinity allows for dynamic reorganization. This lyotropic liquid crystal is then deposited under the action of a shear force (or meniscus force) onto a substrate, so that the shear force (or the meniscus) direction determines the crystal axis direction in the resulting solid crystal film. This shear-force-assisted directional deposition is the third step of ordering, representing the global ordering of the crystalline or polycrystalline structure on the substrate surface. The last fourth step of the cascade crystallization process is drying/crystallization, which converts the lyotropic liquid crystal into a solid crystal film.

The films produced by cascade crystallization process would have a global order. This means that the direction of the crystallographic axis of the film over the entire substrate surface is controlled by the orientation of supramolecular assemblies and by the deposition process, with a limited influence from the substrate surface. Molecules of the deposited material are packed into lateral supramolecular columns with a limited freedom of diffusion or motion.

\section{Conclusion}

With the world market waiting for an energy storage solution, we can conclude that the electrostatic capacitors based on organic molecules will fit this need. We have formulated the requirements for the design of the molecular units which we call dielectrophores. Such units should include at least three subunits, responsible for high polarizability, high resistivity, and high density. We have also outlined the steps of the device fabrication, cascade crystallization, to achieve high-density highly polarizable dielectric thin film with flexible crystalline structure to survive high operating pressure. We believe that the resulting capacitor device would have a specific energy comparable with or surpassing that of, current electrochemical batteries but have other characteristics such as power density, durability, longevity, and so on, far exceeding that of electrochemical batteries.

\section{References}

[1] U.S. Energy Information Administration (EIA) (2016) International Energy Outlook 2016 with Projections to 2040. https://www.eia.gov/outlooks/ieo/pdf/0484(2016).pdf

[2] Hao, X. (2013) A Review on the Dielectric Materials for High Energy-Storage Application. Journal of Advanced Dielectrics, 3, 1330001/1-1330001/14. https://doi.org/10.1142/S2010135X13300016

[3] Conway, B.E. (1999) Electrochemical Supercapacitors: Scientific Fundamentals and 
Technological Applications. Kluwer-Plenum, New York. https://doi.org/10.1007/978-1-4757-3058-6

[4] Shi, Z.Q., Jia, Q.X. and Anderson, W.A. (1991) High-Performance Barium Titanate Capacitors with Double Layer Structure, Journal of Electronic Materials, 20, 939-944. https://doi.org/10.1007/BF02816036

[5] Brown, B., Hess, D., Desai, V. and Deen, M.J. (2006) Dielectric Science and Technology. The Electrochem. Society Interface, 15, 28.

[6] Hartman, R.D. and Pohl, H.A. (1968) Hyperelectronic Polarization in Macromolecular Solids. Journal of Polymer Science Part A-1: Polymer Chemisty, 6, 1135-1152. https://doi.org/10.1002/pol.1968.150060506

[7] Lazarev, P., Lokshin, K. and Nazarov, V. (2001) X-Ray Diffraction by Large Area Organic Crystalline Nano-Films. Mol. Materials, 14, 303-311.

[8] Guselnikov, N., Lazarev, P., Paukshto, M. and Yeh, P. (2005) Translucent LCDs. Journal of Society for Information Display, 13, 339-348. https://doi.org/10.1889/1.1904937

[9] Lehn, J.-M. (1995) Supramolecular Chemistry, Concepts and Perspectives. VCH, Weinheim, 1-10. https://doi.org/10.1002/3527607439

[10] Lydon, J. (1998) Chromonics, in Handbook of Liquid Crystals. Wiley-VCH, Weinheim, Vol. 2B, 981-1007. 